\title{
Reflexiones sobre cultura y política en el área vecina del Caribe
}

Esteban Krotz

...Pensé que si los hombres aprendieran a contarse en el dia los suenos que tuvieron durante toda la noche, podrian hacer de ellos haces de sueños, reivindicaciones de sueños...

Jacques Stephen Alexis, "El rey de los sueños"

Hablar en México de las regiones fronterizas ha significado, hasta hace muy poco, referirse casi exclusivamente a la franja limítrofe con los Estados Unidos y discutir temas tales como la migración de los braceros, la problemática de las maquiladoras y los complejos procesos de aculturación en las ciudades norteñas del pais. Más recientemente, la revolución sandinista, la interminable guerra civil salvadorena, la constante intervención de Washington en Centroamérica y la participación de México en el grupo Contadora han llevado a dirigir mayor atención al sur. Particularmente, el exilio forzado de muchos miles de guatemaltecos, que viven todavia en los campamentos de refugiados en varias zonas de nuestro país y la real o supuesta amenaza a la soberanía nacional proveniente de los conflictos armados en ese país vecino contribuyeron decididamente a aumentar el interés de diferentes sectores sociales $\mathrm{e}$ instituciones públicas y privadas en las circunstancias centroamericanas y la frontera sur de México.' Curiosamente, se debe en parte a esta situación que la tercera frontera mexicana haya quedado prácticamente desapercibida. Por una parte, el término "frontera sur" suele englobar tanto los límites con Guatemala como con Belice, aun cuando este último país suele contarse entre los países caribenos y no entre los centroamericanos. Por otra parte, habitualmente el mar que baña las costas quintanarroense y yucateca es concebido como elemento de delimitación y no de nexo con el mundo caribeño.

A lo anterior se agrega un amplio desconocimiento de los datos y situaciones más elementales del Caribe y la escasa presencia de su problemática en los medios de difusión masiva. ¿Cuántos ciudadanos, periodistas, científicos sociales y políticos conocian la ubicación geográfica, las características sociales y el momento político de Granada, cuando este Estado insular fue invadido -hace ya casi un lustro- por las fuerzas armadas estadunidenses? ¿Dónde puede encontrarse la conciencia de una similitud de problemas culturales determinados entre puertorriqueños y mexica-

\footnotetext{
' Un resumen ilustrativo de la situación que se vincula con las llamadas doctrinas de "seguridad nacional" se encuentra en un artículo de Juan $M$. Sandoval $e t$ al., "La politica de la seguridad nacional y las fronteras de México" en Nueva Antropologia, México, vol. VII, núm. 26, p. 69-175.
} 
nos? ¿Por qué al referirse al litoral oriental de Nicaragua se sigue hablando de una "costa atlántica", como si el mar caribe no existiese?

Los elementos que permitan una mesurada esperanza en que la atención al área vecina del Caribe crezca significativamente en un futuro cercano son pocos. Tal vez el más importante sea la actual lucha del pueblo haitiano para superar los efectos de la larga dictadura duvalierista y para sentar las bases de un nuevo orden social y político. Otro, de carácter marcadamente opuesto, podría ser el repetido intento de Washington de ligar su estrategia militar y politica en Centroamérica con la del Caribe, dando así un sentido particular a la noción de "cuenca del Caribe"." La importancia real de impulsos recientes tales como las visitas del presidente de México a Jamaica y a Belice, ${ }^{3}$ la realización del Primer Festival Internacional de Cultura del Caribe en diversas localidades de Quintana Roo en junio pasado y varios planes para crear una nueva institución académica mexicana especializada en estudios sociales y culturales sobre el Caribe, está todavía por verse.

Las reflexiones que a continuación se presentan ${ }^{4}$ son el resultado de una primera aproximación a esta civilización vecina del Caribe, que a lo largo de la historia ha estado relacionada de muchas maneras con Mesoamérica y con México, especialmente con el sureste del país. Al plantear interrogantes sobre los pueblos y las culturas de este "microcosmos de la humanidad"s y sobre sus perspectivas futuras, se tocan necesariamente también cuestiones clave del análisis sociocultural y del papel que México desempeña en la región.

Parece que cuando se habla de las culturas caribeñas, se da con frecuencia un fenómeno bien documentado en cierta literatura antropológica acerca de fenómenos culturales y, en especial, de las llamadas culturas populares. Particularmente cuando no se trata de expresiones culturales escritas (tales como literatura, poesia, teatro), la palabra se asocia ante todo a una serie de hechos y con-

\footnotetext{
2Recientemente, dos autores esbozan un breve panorama al respecto: Pablo A. Martinez, "El Caribe, cuatro años después de la ocupación militar de Granada" en $E l$ Caribe Contemporáneo, México, 1987, núm. 15, p. 22-29 e Ilya Villar, "Crisis y geopolítica" en El Caribe Contemporáneo, 1987, núm. 15, p. 33-42.

${ }_{3}$ El discurso del presidente mexicano ante el parlamento jamaiquino se encuentra en el número 15 de la revista El Caribe Contemporáneo (p. 101-104). La visita a Belice se realizó en mayo de 1988 y fue la primera visita oficial de un presidente mexicano a esta nación independizada en 1981. En este contexto también merece mención la exposición temporal "Belice: muchas culturas, una nación", inaugurada a fines de 1987 en el Museo Nacional de las Culturas de la ciudad de México.

+ El presente ensayo se basa en la ponencia "Escuchar y hacer escuchar la palabra de una civilización vecina", presentada en el Foro sobre identidad cultural y culturas populares en el Caribe, que se realizó del 10 al 15 de junio de 1988 en el marco del Primer Festival Internacional de Cultura del Caribe en la ciudad de Cancún.

${ }_{5}^{5}$ Asi ha calificado sugerentemente Gérad Pierre-Charles a la región caribeña en su "Microcosmos de la humanidad" en Cuadernos del Tercer Mundo, México, 1979 , núm. 30 , p. 12-17; en un estudio más reciente ha elaborado la noción de "Civilización", al respecto véase El pensamiento sociopolitico moderno en el Caribe, México, FCE, 1985, p. 248 y ss.
} 
ductas de alguna manera llamativos, por no decir "exóticos". Es decir, ocupan un lugar privilegiado elementos tales como indumentaria, tecnologia, artesanías, morada, fiestas, a veces la música y el baile, también la estructuración y reproducción de determinadas funciones y relaciones sociales en el nivel familiar y "comunitario". Es pertinente destacar que estos elementos son básicamente observados, al menos observables; quiere decir, de algún modo "externos", "de superficie". En consecuencia puede verse una llamativa reducción de hecho del concepto de cultura en general y de la cultura popular en especial de artefactos y conductas que, a su vez, fundamentan la necesidad del antropólogo como intermediario entre los estudiados y otros sectores sociales. Y también por eso, las culturas -de México, del Caribe; populares o noestán siempre al borde la cosificación exteriorizada: petrificadas como en los museos comunes, o más movidas como en algún tipo de show folclórico. Y también por eso hasta a los antropológos nos cuesta trabajo a veces reparar en que la cuestión de la cultura tiene que ver siempre también con esta dimensión -aun tratándose de expresión y asociación de ideas, con criterios para la evaluación de hechos y acontecimientos, con modelos de concebir la naturaleza humana y extrahumana y de interactuar con ella, con sistemas de valores y pautas afectivas, con modos de comunicación, de legitimación y deslegitimación, con propuestas para dar sentido al dolor, al deseo, al logro, al fracaso, con sueños que se dirigen hacia el futuro, pero que al mismo tiempo orientan la apreciación del presente, con decisiones y justificaciones sobre formas de organizar las relaciones entre los sexos y las generaciones, las de tipo laboral y las políticas. Sólo la conciencia de tener que ver siempre también con esta dimensión -aun tratándose de artefactos y conductas- es capaz de fundamentar, cuando se habla de "otras" culturas en cualquier parte del universo, un acercamiento a ellas, donde el estudioso reconoce en los estudiados sujetos iguales a sí mismo y en su cosmovisión algo que puede interrogar y complementar la suya propia. Este es el tipo de acercamiento a las culturas del Caribe y los pueblos que las han creado y las viven, que es necesario para que la vecindad geográfica pueda ser la base de un intercambio enriquecedor en todos los órdenes y para que la investigación social y la difusión de sus resultados tenga perspectivas prometedoras para todos los involucrados en ellas.

Una de las preocupaciones más frecuentes en la discusión actual sobre las culturas populares en toda América Latina -y en este aspecto tampoco veo razón por la cual la cuenca del Caribe no tenga que ser vista como parte de nuestro llamado "subcontinente"- suele hacer hincapié en el creciente peso de la estandarización impositiva a que están sujetas. En efecto, la cocacolanización, ${ }^{6}$ conjunto de tendencias homogeneizantes difundidas con

' El término ha sido tomado de un ensayo de Thierry Verhelst sobre la crisis cultural de Occidente. Véase su "The Cultural Crisis and Oportunities of the West and International Solidarity" en Ifda dossier, Nyon, núm. 65, p. 51-66. 
fuerza desde los centros de la organización capitalista industrialista de la vida contemporánea, ha hecho desaparecer muchos elementos culturales tradicionales y mercantilizado otros tantos. Esto vale tanto para los niveles más visibles del vestido, la comida, los utensilios caseros, aspectos de la organización del trabajo y del ocio como para las estructuras y los componentes de la cosmovisión en el sentido explicitado hace un momento.

Pero también aquí es cierto que cualquier fuerza social produce su propia contradicción y es precisamente en los países más industrializados y centralmente organizados donde se suceden constantemente olas de expresiones contraculturales y surgen por todos lados movimientos que impugnan elementos claves del desorden social establecido. Me parece, por lo tanto, que el rescate, la conservación y la revaluación de elementos tradicionales de las culturas en el sentido amplio que se acaba de esbozar, constituyen tareas vigentes y urgentes, porque cada cultura que se extingue implica la pérdida para siempre de una parte del acervo de impulsos para seguir creando y recreando el mundo. Pero más importante me parece la atención a los elementos de resistencia, impugnación y propuesta alternativa que se pueden vislumbrar especialmente en las culturas populares. Porque éstas son (cosa que a veces se pierde en la discusión académica y hasta política), las formas de vida de aquellos que mayoritariamente no tienen, precisamente a diferencia de quienes trabajamos "sobre ellos", resueltas las necesidades básicas propias y de los suyos. Es patente que esta propuesta no puede considerarse una idea particularmente original, ya que la influencia gramsciana en la antropología latinoamericana ha contribuido a reforzar, aunque no sin malentendidos neorománticos, este enfoque en los últimos años. ${ }^{7} \mathrm{Em}$ pero, dista todavía de ser aceptada de hecho en muchos estudios y en la reflexión cotidiana. Así, por ejemplo, la más breve ojeada a los estudios históricos y socioantropológicos del área caribeña nos muestran una multifacética tradición rebelde, pero pocas veces se le tiene presente fuera de ella. $O$ ¿quién piensa en Venezuela y México cuando se habla de la lucha independiente, quien en los protagonistas haitianos de la primera revolución anticolonial de la región, quién conoce sus nombres, recuerda las fechas? ¿Quién se ha acercado a la historia de los piratas, cimarrones, garifunas, a las revueltas de esclavos, a expresiones artísticas y religiosas desde esta perspectiva? ¿No es cierto que incluso la Revolución cubana, hace ya casi una generación fundamento de las más amplias esperanzas, impulso para imaginaciones audaces y acciones no menos osadas, ha sido mucho más opacada de lo que pudiera resultar del desgaste natural de cualquier experimento social? No se propone aquí la realización de estudios impregnados de ideología -aunque sea desde otra perspectiva- como la mayor parte de la historiografia y las ciencias sociales establecidas, sino un acercamiento a los fenómenos socioculturales del pasado y del presente que parta del interés de descubrir en ellos los impulsos objetivos y subjetivos

\footnotetext{
${ }^{7}$ Véanse para esto las consideraciones de Alberto M. Cirese, especialmente el ensayo "Intelectuales, folclor, instinto de clase", en Ensayos sobre las culturas subalternas, México, Casa Chata, 1979.
} 
que apuntan utópicamente a un mundo radicalmente diferente. ${ }^{8}$ Así, precisamente el área caribeña parece tener una importancia especial para mantener por doquier la convicción siempre amenazada de que ningún fracaso es suficientemente fuerte para cancelar los sueños de una vida libre y digna que se desarrolla en un ambiente de justicia y de intercambio enriquecedor entre los individuos y las colectividades. Atención especial merecerian aqui probablemente las circunstancias en las que elementos meramente opositores despliegan su dimensión alternativa en la realización -germinal, inicial, fragmentaria- de este mundo nuevo.

En el Caribe es particularmente visible lo que también vale para toda la América Latina: la estrecha vinculación entre cultura y poder. Desde sus inicios como civilización de las Indias Occidentales hace medio milenio, hasta la actualidad, desde el aspecto étnico racial hasta el religioso y lingüistico, tenemos que ver aquí con productos de una doble correlación de fuerzas: la de las potencias colonialistas entre sí, por una parte, y por otra la que se daba entre el peso moldeador del dominador colonial y la resistencia pasiva o activa del dominado. Reconocer este hecho no significa, a mi modo de ver, una indebida politización de la cuestión cultural, ni su liga necesaria a proyectos políticos específicos. ${ }^{9}$ Pero independientemente de una definición precisa de características y límites de la civilización antillana e independientemente también de la problemática teórica y práctica de los diversos aspectos relacionados con las culturas populares del área, me parece que hay un punto indiscutible. No es imaginable un futuro digno de tal nombre para esta civilización ni para las colectividades que la componen si sigue siendo la mayor área colonial del mundo contemporáneo. No importan las construcciones jurídicas con las que se esté disfrazando el hecho. Es cierto, además, que aun siendo

\footnotetext{
* Para un acercamiento a esa perspectiva puede verse el trabajo de Esteban Krotz, Utopía, 2a. ed., México, UAM-Iztapalapa, 1988.

" La antropóloga Amalia Signorelli ha resumido esta cuestión así: "En la sociedad contemporánea no existe realidad cultural alguna (porque no existe realidad social alguna) que pueda ser analizada en su actualidad fuera del marco general de las relaciones de dependencia entre áreas centrales y áreas marginales, entre áreas de concentración de capitales y tecnologia, y áreas periféricas de subdesarrollo, entre áreas del poder y áreas de explotación. Es claro que en este caso el término 'área' no indica sólo un lugar geográfico, sino que también remite siempre a un lugar social, a una relación entre clases sociales. No hace falta insistir largamente sobre el carácer planetario y omnipresente de la relación desarrollo-subdesarrollo, pero el reconocimiento de su existencia impone que las investigaciones antropológicas sobre la realidad actual ya no puedan justificarse de ningún modo como investigaciones etnológicas sobre los primitivos o como investigaciones folklóricas sobre los campesinos, entendiendo a unos y a otros como realidades autónomas y separadas: es cierto que cuando se examina la realidad actual, ya no se tiene enfrente nada que no sea interno a las relaciones de dependencia entre centro y periferia, entre dominantes y dominados, entre metrópoli hegemónica y territorio subalterno. En este sentido, la antropologia actual sólo puede ser de ahora en adelante... una antropología de las saciedades complejas, porque no puede no ser una antropología de las relaciones de dominación y de los conflictos que derivan de ellas." Veáse "Antropología, culturología, marxismo" en G. Giménez (comp. 1), La teoria y el análisis de la cultura, Guadalajara, Universidad de Guadalajara, 1987. En este mismo texto también se llama la atención sobre malas interpretaciones ingenuas.
} 
toda la región formalmente independiente de sus antiguos amos coloniales, no se eliminará automáticamente ni la dependencia imperialista general a la que se encuentra sometida toda América Latina, ni se anularán las influencias de empresas y bancos transnacionales, de pactos militares impuestos, de la narcomafia, y que tampoco se solucionarán de un golpe las contradicciones internas de cada una de estas colectividades. Pero tampoco se enfrentará a ninguno de estos retos sin salirse de la clasificación formal -politica, jurídica- de tratarse de sociedades y de personas definitivamente de segunda, distinción que difícilmente puede ser concebida como estímulo para cualquier tipo de desarrollo social y de creación cultural promisorio.

Estamos, como es ampliamente sabido, bajo la sombra de un suceso que a falta de una denominación más o menos libre de implicaciones ideológicas y políticas que ya han provocado airadas polémicas, se ha convenido en llamar "El Quinto Centenario". Queramos o no, en los próximos años la atención pública mundial, especialmente la europea y la norteamericana, se va a fijar con más detenimiento precisamente en el Caribe. Me parece que deberiamos aprovechar esta situación para un recordatorio y para una exigencia. El recordatorio de que el Caribe pagó en una altísima proporción con vidas humanas y recursos naturales el despegue de la industrialización del mundo moderno y la hasta el día de hoy prevaleciente posición privilegiada de la mayor parte de los países ricos. ${ }^{10}$ El recordatorio, en consecuencia, también, de que la pobreza y la angustia en esta parte del mundo -identificada en Europa y Norteamérica principalmente por los carteles paradisiacos de las agencias de viaje y por determinados ritmos musicales- es resultado no de la buena vida de sus habitantes tropicales, sino de su expoliación secular por quienes lo visitan ahora masivamente como turistas. Y la exigencia, que podria resumirse así: „Por un Quinto Centenario sin colonias! Se trata, simplemente, de no conformarse con una intolerable lesión de los derechos humanos -individuales y colectivos- que implica una grave hipoteca para cualquier desarrollo armónico de las personas y de los pueblos. Sin ser condición suficiente para el desarrollo futuro con dignidad y libertad de esta región, es condición necesaria la abolición definitiva de todas las colonias en el Caribe.

Uno de los aspectos principales que se ha discutido una y otra vez en torno a la independencia de los países caribeños, ha sido el de su viabilidad económica (y tanto la perspectiva como la experiencia parecen demostrar su inviabilidad en términos del sistema capitalista vigente). Menos parece haberse cuestionado su viabilidad política -a tal grado que la necesidad de una organización estatal con su constitución, sus tres poderes, sus elecciones, etc., se ha exigido y propuesto casi siempre como modelo casi natural y como garantía para una vida colectiva promisoria-. Allí también

${ }^{10}$ Sidney W. Mintz ha dado énfasis a este punto. Veáse "The Caribbean as a Socio-Cultural Area" en M. H. Horowitz (ed.), Peoples and Cultures of the Caribbean, Nueva York, Natural History Press, 1971, p. 17-46. 
debe radicar la concepción de la existencia y la necesidad de una "cultura nacional", reforzada aquí por una situación en la que muchos pueblos caribeños aparecen como "hijos de la geografia" aparentemente sin alternativa. ${ }^{12}$

¿Será posible mantener hoy todavía, en toda la extensión de la palabra, la noción de la "soberanía nacional" como base y como meta de la organización colectiva? ¿Será posible presentar la necesidad o existencia de una cultura nacional en este momento, cuando ni siquiera los países, que originalmente nacieron bajo los reclamos de las primeras burguesías por un Estado nacional mantienen esta idea de soberanía, ni las ficciones ideológicas correspondientes? ¿Será posible seguir señalando la creación y el reforzamiento de Estados-naciones como camino viable?

A mi modo de ver, el Caribe demuestra hace tiempo lo que en muchos lugares del mundo industrializado se empieza a sospechar: el tiempo ha madurado para pensar intensivamente en otras formas de organización sociopolítica. Y esto es -independientemente de cualquier otra cosa- también un problema cultural. ${ }^{13}$ Porque es un problema de la imaginación y del análisis, del debate teórico y de la inventiva, del estudio y de la prospectiva; es, a riesgo del malentendido, un problema del ejercicio utópico. ¿No será el Caribe -precisamente por su carácter sociohistórico general- su ambiente ideal? Es cierto que pocos científicos norteamericanos o europeos esperarían mucha originalidad de planteamiento y de soluciones provenientes de esta área como, en términos generales, de ninguna parte de América Latina. Pero lo peor es que muchos latinoamericanos -y aquí incluyo a los caribeños- han interiorizado este "racismo intelectual". ${ }^{14}$ Obviamente, no se trata aquí de defender el ensayo superficial tan frecuente en las ciencias sociales latinoamericanas en aras de una supuesta originalidad, que en una disciplina científica necesitará siempre del

" René Zavaleta Mercado, "Notas sobre la cuestión nacional en América Latina" en J. E. Vega (coord.), Teoria y política de América Latina, 2a. ed., México, Centro de Investigación y Docencia Económica, 1988, p. 281-289.

12 Releer la obra del gran antillano Frantz Fanon, Los condenados de la tierra, México, FCE, 1965, hace comprensible la reclamación de un Estado nacional independiente y la insistencia en la cultura nacional como opuesta a la de los colonizadores. Por otra parte, parece existir una llamativa coincidencia entre las diversas posiciones políticas actuales sobre el futuro político de Puerto Rico y el debate anterior sobre la misma cuestión en la República Dominicana, resentado por $G$. Pierre-Charles, El pensamiento..., op. cit., p. 153 y ss. Pero precisamente la realidad sociopolítica, militar, económica y cultural de los Estados-naciones antillanos independizados hace mucho o poco parece indicar la necesidad de planteamientos realmente alternativos, planteamientos que en un sentido nuevo permiten "que la imaginación se libere del orden colonial".

${ }^{13}$ En este contexto es pertinente recordar la observación de Jean Casimir hecha en el mencionado foro (véase nota 4), en el sentido de que cultura y organización social populares han sido forjados frecuentemente en contra de las estructuras estatales vigentes, mismas que suelen arrogarse la representación de "lo nacional".

${ }^{14}$ Rex Nettleford, "La acción cultural y el cambio social" en A. Brown (comp.), La irrupción del Caribe, México, Méx-Sur, 1984, p. 50 y ss., han hecho un vigoroso alegato en este sentido y recientemente $M$. Vargas Llosa ha denunciado en una entrevista este "nuevo racismo del siglo XX", en que intelectuales y políticos de los paises metropolitanos fundan su escepticismo acerca de la capacidad creadora de Latinoamérica en los campos de la cultura y la política. Veáse "Demokratie hat zu wenig Sex-Appeal" en Der Spiegel, Hamburgo, vol. 41, núm. 49, p. 167-182. 
trabajo arduo y colectivo. Pero, por una parte, la realidad social y política propia del Caribe proporciona un impulso vital para la búsqueda intensa de nuevas formas de convivencia organizada e incluso institucional. $Y$, por otra, la misma tradición caribeña parece llena de reflexiones teóricas y de intentos prácticos que apuntan hacia esta dimensión. Precisamente el último hecho subraya la necesidad urgente de la recuperación de los intentos de análisis social formulados una y otra vez por pensadores antillanos $y$, de igual manera, de los elementos de análisis y de propuesta contenidos generalmente sin sistematización y con reducido grado de explicitación en tantos movimientos populares testimoniados en la región -no para hacer otra antología de textos, provista de una docta introducción del recopilador-, sino como estímulo para asumir una realidad y una tradición que pueden servir como base para una aportación al debate de los problemas sociales cruciales de nuestro tiempo y a la solución práctica de las necesidades vitales -tanto fisiológicas como culturales- de los pueblos latinoamericanos. $^{15}$

No se necesita mucha perspicacia para suponer que en el futuro se repetirán reuniones de científicos, intelectuales y artistas antillanos y/o especialistas externos interesados en el Caribe. Lo mismo vale para las reuniones de gerentes de cadenas hoteleras transnacionales, funcionarios de las administraciones públicas $y$ oficiales de los ejércitos de la región y de personajes similares. Por ello es pertinente considerar aquí y adecuar una propuesta que durante los últimos años, al parecer, ha crecido en fuerza. Como complemento a la actual Asamblea General de las Naciones Unidas, que es una reunión de representantes gubernamentales, ha sido formulado un modelo para reunir en una especie de "Cámara Baja" a representantes de los pueblos cuyos Estados componen esta organización mundial. ${ }^{16}$ ¿No seria deseable y posible contar con una asamblea de representantes de los pueblos caribeños, cuyo horizonte vaya más allá que el de sus representantes gubernamentales $y / o$ de sus científicos (incluyendo a los especialistas en ciencias sociales y humanidades, quienes a menudo se arrogan de manera implícita una especie de representatividad no cubierta por ningún mandato formal ni informal)? Se trataría de una reunión que -al margen de proyectos partidistas específicos o de problemas nacionales particulares- funcionaria como caja de resonancia para la expresión de necesidades prácticas y de problemas teóricos, para la comunicación de avances en el conocimiento y la discusión de alternativas, para la propuesta de modelos generales y de estrategias coyunturales, para el intercambio de cosmovisiones globales y de ideas particulares, como estimulo para la creación y la inventiva, como foro permanente para la formación y aplicación de criterios sobre los mecanismos y las metas del desarrollo

15 He tratado de precisar esta necesidad en un ensayo reciente. Esteban Krotz, Historia e historiografia de las ciencias sociales en América Latina y el Caribe: consideraciones introductorias a la problematica. Ponencia presentada en Amsterdam, en el $46^{\circ}$ Congreso Internacional de Americanistas.

16 El resumen de esta proposiciôn se encuentra en Ifda dossier, núm. 64. 
de la región que corresponda a las aspiraciones de todos sus habitantes.

Estas reflexiones sobre el Caribe se han referido al conceptualismo del hecho cultural y operatividad de sus análisis y a algunos aspectos concretos de la convivencia de los pueblos del archipiélago antillano y de la región que lo circunda. Es de desearse que el redescubrimiento de la tercera frontera de México, la frontera caribeña, vaya a al par con la construcción de un nuevo concepto de frontera. Esta construcción, tarea teórica y práctica al mismo tiempo, tendría que concebir "frontera" no en el sentido tradicional de una linea que divide y que es ante todo un asunto de controles, prohibiciones, guardias y militares, sino más bien en el sentido de una región que pone en contacto, que relaciona, que une ${ }^{17}$ y que es, por consiguiente, algo que tiene que ver con invitación, participación, intercambio y compenetración. Un enfoque de este tipo llevaria también al reconocimiento de que México -al menos, algunas de sus partes- ha estado vinculado a lo largo de toda su historia, incluso antes de la llegada de los conquistadores ibéricos, con las culturas antillanas y, en consecuencia, a una comprensión más cabal de su historia e identidad. Por otra parte permitiria pensar con más amplitud el futuro de este mosaico multifacético, que es Latinoamérica, donde sigue siendo un problema político y cultural de primer orden la tentación siempre renovada de convertir la diferencia entre las configuraciones socioculturales en punto de partida para tácticas subimperialistas de todo tipo en vez de en elemento central para un desarrollo individual y colectivo auténticamente democrático y plural.

\section{Bibliografía}

Alexis, Jacques Stephen, "El rey de los sueños", Plural, México, vol. XVII, núm. 181, 1985, p. 8-13.

Cirese, Alberto M., Ensayo sobre las culturas subalternas, México, Casa Chata, 1979.

Fanon, Frantz, Los condenados de la tierra, 2a. ed., México, Fondo de Cultura Económica (FCE), 1965.

"For a United Nations Second Assembly: Summary of a Proposal", Ifda dossier Nyon, núm. 64, 1988, p. 57-58.

Krotz, Esteban, Utopia, 2a. ed., México, UAM- Iztapalapa, 1988.

- Historia e historiografia de las ciencias sociales en América Latina y el Caribe: consideraciones introductorias a la problemática. Ponencia presentada en el $46^{\circ}$ Congreso Internacional de Americanistas, Amsterdam, 1988.

Mariñez, Pablo A., "El Caribe, cuatro años después de la ocupación militar de Granada", El Caribe Contemporáneo (México), 1987, núm. 15, p. 22-29.

Mintz, Sidney W., "The Caribbean as a Socio-Cultural Area" en M. H.

\footnotetext{
${ }^{17}$ Ideas importantes acerca de esta concepción se encuentran en la contribución de Jan de Vos al mencionado foro (véase nota 4), cuya memoria se encuentra en preparación.
} 
Horowitz (ed.), Peoples and Cultures of the Caribbean, Nueva York, Natural History Press, 1971, p. 17-46.

Nettleford, Rex, "La acción cultural y el cambio social" en A. Brown (comp.), La irrupción del Caribe, México, Mex-Sur, 1984, p. 41-100.

Pierre-Charles, Gérard, "Microcosmos de la humanidad", Cuadernos del Tercer Mundo, México, núm. 30, 1979, p. 12-17.

- El pensamiento sociopolítico moderno en el Caribe, México, FCE, 1985.

Sandoval, Juan M., Francisco J. Guerrero y Ma. Eugenia del Valle, "La política de la Seguridad Nacional y las fronteras de México", Nueva Antropologia, México, vol. VII, núm. 26, 1985, p. 69- 175.

Signorelli, Amalia, "Antropología, culturología, marxismo" en G. Giménez M. (comp.), La teoria y el análisis de la cultura, Guadalajara, Universidad de Guadalajara, 1987, p. 205-206.

Vargas Llosa, Mario, "Demokratie hat zu wenig Sex-Appeal" (entrevista), Der Spieggel, Hamburgo, vol. 41, núm. 49, 1987, p. 167-182.

Verhelst, Thierry, "The Cultural Crisis and Opportunities of the West and International Solidarity", Ifda dossier Nyon, núm. 65, 1988, p. 51-66.

Villar Martínez, Ilya, "Crisis y geopolitica en el Caribe" en El Caribe Contemporáneo, núm. 15,1987, p. 33-42.

Zavaleta Mercado, René, "Notas sobre la cuestión nacional en América Latina" en J. E. Vega (coord.), Teoría y política de América Latina, $2 \mathrm{a}$. ed., México, Centro de Investigación y Docencia Económica, 1984, p. 281-289. 